Published in final edited form as:

J Hypertens. 2009 April ; 27(4): 709-711. doi:10.1097/HJH.0b013e3283295dd4.

\title{
Cerebral microcirculation in hypertension
}

\author{
Guido Grassia,b and Donald D. Heistad ${ }^{\mathrm{c}}$ \\ ${ }^{a}$ Clinica Medica, Dipartimento di Medicina Clinica e Prevenzione, Università Milano-Bicocca, \\ Ospedale San Gerardo, Monza \\ 'Istituto Auxologico Italiano, Milan, Italy \\ ${ }^{\circ}$ Cardiovascular Center, Departments of Internal Medicine and Pharmacology, University of lowa \\ Carver College of Medicine, lowa City, lowa, USA
}

\section{Keywords}

cerebral arterioles; hypertension; microcirculation; myography

\begin{abstract}
In the present issue of the journal, Rizzoni et al. [1] publish an interesting study reporting for the first time the structural as well as functional alterations of cerebral arterioles in human hypertension. By using the micromyographic technique applied to biopsies of cerebral tissue obtained during neurosurgery, the authors provide three findings. First, they show that the media-to-lumen ratio of cerebral arterioles is greater in hypertensive patients as compared with age-matched normotensive controls. Second, they provide evidence that cerebral cortical microvessel density is markedly reduced in patients with high blood pressure. Finally, Rizzoni et al. [1] document that the mechanical and microstructural properties of the cerebral arterioles, assessed via the strain-stress curves and the collagen microvessel wall content, respectively, are similar at normal and high levels of blood pressure. The authors conclude that the cerebral microcirculation undergoes profound structural modifications in the hypertensive state, without, however, concomitant alterations in arteriolar stiffness and in the fibrotic composition of the vessel wall. All of these structural alterations speak in favour of the so-called eutrophic vascular remodelling previously described in experimental animals [2].
\end{abstract}

The results of the present study offer new insights in the pathophysiology of hypertensive cerebral vascular disease. They also raise, however, several questions of both methodological and clinical relevance. Three of these questions will be discussed in the present editorial commentary, focusing in particular on what is the background knowledge for the present study, how do the new data fit with the well established pathophysiological picture of the cerebrovascular alterations provided by previous investigations and the study strengths and limitations.

Correspondence to Professor Guido Grassi, Clinica Medica, Ospedale S. Gerardo dei Tintori, Via Pergolesi 33, 20052 Monza, Milan, Italy Tel: +39 039233 357; fax: +39 039322 274; email: guido.grassi@ unimib.it.

Editorial comment

Remodelling of small cerebral arteries in human hypertension: structural and functional alterations 


\section{Cerebral microvascular structural alterations in hypertension}

It is a well established notion that cerebral blood flow remains almost unaltered in its main functional patterns in essential hypertension. The only documented alterations in established hypertension are a shift to the right of the pressure/flow relationship in a way that cerebral blood flow does not increase in face of a sustained blood pressure elevation [3].

Despite the lack of substantial strain-stress functional modifications, structural cerebrovascular changes do occur in hypertension, as indicated by data collected in various experimental animal models [4]. These alterations are so marked and widespread as to lead to a reduction in the external diameter of the vessel, and thus to encroachment of the vessel wall on the lumen of the cerebral arterioles [5]. These structural arteriolar alterations, which include vascular hypertrophy, have a major impact on cerebrovascular circulation, being largely responsible for the impairment of the cerebral vasodilatation that takes place in hypertension [5]. Our knowledge of hypertension-induced structural alterations of the cerebral circulation has been recently expanded with evidence that middle cerebral arteries of spontaneously hypertensive rats display a reduction in their lumen-diameter ratio across the entire blood pressure range, confirming once more the occurrence of eutrophic inward remodelling [6].

The above-mentioned structural alterations of cerebral blood vessels that occur in hypertension trigger both favourable and unfavourable consequences. Among the former, three deserve a special mention. First, vascular hypertrophy and remodelling trigger an increase in wall thickness and reduction of the vascular diameter, leading to a virtual normalization of the vascular wall stress [7]. Secondly, the above-mentioned vascular changes protect the cerebral circulation in hypertension, by attenuating the increase in cerebral microvessel pressure, as well as its deleterious effects on microcirculation [8]. Finally, structural alterations of the cerebral microcirculation participate in the abovementioned cerebral blood flow autoregulatory process $[5,9]$.

Cerebral vascular hypertrophy and remodelling also exert unfavourable consequences. These include encroachment on the lumen of the cerebral arterioles, as already described, as well as impairment of the maximal vasodilatatory responses to a variety of stimuli, such as hypercapnia, hypoxia and endothelium-mediated vasodilatation [10]. Recent data by Izzard et al. [6], however, have modified these notions by showing that the structural/functional relationships in the cerebral microcirculation may be, at least in part, age-dependent and that the reduction in arterial distensibility may be a phenomenon typical of the more advanced stages of the hypertensive state, whereas eutrophic inward remodelling may occur in an earlier phase of the disease. This may confirm in cerebral vessels the evidence provided some years ago that remodelling of small arteries may represent the earliest alteration of the vascular tree in hypertension [11].

\section{Merging together old and new evidence}

The study by Rizzoni et al. [1] documents the occurrence in humans of microvascular alterations observed years ago in experimental animal models of hypertension. They demonstrated that the media-to-lumen ratio is increased when blood pressure levels are 
chronically elevated. The present study adds two sets of new data to this information, however. The first one is microvessel rarefaction, detected as in the present study. The second one is the lack of differences between hypertensive and normotensive people as far as collagen composition of the vessel wall is concerned, which indicate the absence of an early or advanced fibrotic process. The data per se may fit well with the previously mentioned finding that in animal models of hypertension cerebral vascular distensibility is increased as compared with the normotensive state [5,6]. Composition of the vessel wall, however, may be different according to the pathophysiological mechanisms responsible for the development and progression of the hypertensive state. For example, in cerebral arterioles of renal hypertensive rats, the normodistensible-to-distensible components ratio was less in renal hypertensive arterioles than in normotensive ones [12]. This likely depends on the profibrotic effects exerted by angiotensin II, whose circulating and tissue levels are markedly increased in the hypertensive state secondary to renal artery stenosis [13,14]. It is unlikely that the behaviour of the fibrotic component observed in hypertensive patients in the present study was affected in some way by antihypertensive drug treatment, because data subanalysis did not show differences between different drug treatments, even when comparison was made with drugs acting on the renin-angiotensin-aldosterone system [1].

\section{Strengths and limitations of the study}

The major strength of the study [1] is the use of the micromyographic technique in humans to obtain information on effects of hypertension on structure of the cerebral microcirculation. Another study strength is represented by the assessment of the microvessel density in the cerebral tissue performed via an automated imaging analysed approach.

The study is not exempt from limitations, however. The first, and probably the most important, limitation is that the cerebral tissue sample for the investigation of the microvessels was taken from normotensive and hypertensive patients during a neurosurgical intervention for benign or malign tumour. As the tissue sample, by necessity, was close to the neoplastic area, one cannot exclude the possibility that changes in the vascular structure around the tumour or the effects of neoplastic tissue-related substances or both (e.g. cytokines) may have modulated the main study results. The authors, however, correctly point out that if this was the case, the confounding effects probably should have affected normotensive as well as hypertensive microcirculation. Another limitation of the study, which is common to all micromyographic investigations, is that the data obtained in a single blood vessel cannot be safely extrapolated to other vascular regions. It is thus impossible to know whether the microvascular alterations occurring in hypertension are homogeneous or heterogeneous in humans. In addition, the time course of the hypertension-related microvascular alterations occurring in different circulatory districts is not clearly defined. In particular, it still remains to be determined whether the observed alterations in some regional microcirculations may occur at earlier phase than those characterizing other vascular beds. This information could be of great clinical value by allowing therapeutic interventions to be directed toward specific vascular regions. 


\section{References}

1. Rizzoni D, De Ciuceis C, Porteri E, Paiardi S, Boari GEM, Mortini P, et al. Altered structure of small cerebral arteries in patients with essential hypertension. J Hypertens. (in press).

2. Mulvany MJ, Aalkjaer C. Structure and function of small arteries. Physiol Rev. 1990; 70:921-961. [PubMed: 2217559]

3. Strandgaard S, MacKenzie ET, Sengupta D, Rowan JO, Lassen NA, Harper AM. Upper limit of autoregulation of cerebral blood flow in the baboon. Circ Res. 1974; 34:435-440. [PubMed: 4363762]

4. Baumbach GL, Heistad DD. Remodeling of cerebral arterioles in chronic hypertension. Hypertension. 1989; 13:968-972. [PubMed: 2737731]

5. Baumbach GL, Heistad DD. Adaptive changes in cerebral blood vessels during chronic hypertension. J Hypertens. 1991; 9:987-991. [PubMed: 1661770]

6. Izzard AS, Horton S, Heerkens EH, Shaw L, Heagerty AM. Middle cerebral artery structure and distensibility during developing and established phases of hypertension in the spontaneously hypertensive rat. J Hypertens. 2006; 24:875-880. [PubMed: 16612249]

7. Ibrahim J, Berk BC. Flow-mediated vascular remodeling in hypertension. Relation to hemodyamics. Stroke. 2008 [Epub ahead of print].

8. Werber AH, Heistad DD. Diffusional support of arteries. Am J Physiol. 1985; 248(6 Pt 2):H901H906. [PubMed: 4003567]

9. Folkow B, Hallbäck M, Lundgren Y, Sivertsson R, Weiss L. Importance of adaptive changes in vascular design for establishment of primary hypertension, studied in man and in spontaneously hypertensive rats. Circ Res. 1973; 32(Suppl 1):2-16. [PubMed: 4576385]

10. Baumbach GL, Heistad DD. Cerebral circulation in chronic arterial hypertension. Hypertension. 1988; 12:89-95. [PubMed: 3044994]

11. Park JB, Schiffrin EL. Small artery remodeling is the most prevalent (earliest?) form of target organ damage in mild essential hypertension. J Hypertens. 2001; 19:921-930. [PubMed: 11393676]

12. Baumbach GL, Hajdu MA. Mechanics and composition of cerebral arterioles in renal and spontaneously hypertensive rats. Hypertension. 1993; 21:816-826. [PubMed: 8500863]

13. Mehta PK, Griendling KK. Angiotensin II cell signaling: physiological and pathological effects in the cardiovascular system. Am J Physiol Cell Physiol. 2007; 292:C82-C97. [PubMed: 16870827]

14. Javeshghani D, Sairam MR, Neves MF, Schiffrin EL, Touyz RM. Angiotensin II induces vascular dysfunction without exacerbating blood pressure elevation in a mouse model of menopauseassociated hypertension. J Hypertens. 2006; 24:1365-1373. [PubMed: 16794486] 